\title{
ACKNOWLEDGMENT OF REVIEWERS
}

The success of Molecular Plant-Microbe Interactions depends on the quality of manuscripts submitted by authors and on the care and competence with which they are reviewed. It is the policy of the Editorial Board to solicit reviews of manuscripts from specialists most qualified to review them. In addition to members of the Editorial Board, the individuals listed below provided constructive critical reviews of one or more manuscripts during the past year. Their names are published here in grateful appreciation for their contributions to the journal.

T. Adams, Texas A\&M University, College Station, TX, U.S.A.

C. Allen, University of Wisconsin, Madison, WI, U.S.A.

A. J. Anderson, Utah State University, Logan, UT, U.S.A.

J. Anderson, USDA Weed Science Laboratory, Beltsville, MD, U.S.A.

S. Angell, John Innes Centre, Nowich, England

J. Antoniw, Rothamsted Experimental Station (IACR), Harpendent, United Kingdom

M. Arlat, CNRS-INRA, Castanet-Tolosan, France

S. J. Barker, University of Adelaide, Waite-Osmond, Australia

O. W. Barnett, North Carolina State University, Raleigh, NC, U.S.A.

F. Barras, LCB - CNRS, Marseille, France

T. Baskin, University of Biological Sciences, Columbia, MO, U.S.A.

W. D. Bauer, Ohio State University, Columbus, OH, U.S.A.

C. Bender, Oklahoma State University, Stillwater, OK, U.S.A.

A. Bent, University of Illinois, Urbana, IL, U.S.A.

A. Bhagwat, USDA-ARS, Soybean and Alfalfa Research Laboratory, Beltsville, MD, U.S.A.

T. Bisseling, Wageningen Agricultural University, Wageningen, The Netherlands

D. Blevins, University of Missouri, Columbia, MO, U.S.A.

J. Bol, Leiden University, Leiden, The Netherlands

P. Bottomley, Oregon State University, Corvallis, OR, U.S.A.

N. Brewin, John Innes Centre, Norwich, United Kingdom

W. Broekaert, F A Jenssenslab Voor Genetica, Leuven, Belgium

C. R. Bronson, Iowa State University, Ames, IA, U.S.A.

S. Brumbley, Bureau of Sugar Experiment Stations, Indooroopily, Australia

R. Buell, Carnegie Institute, Stanford, CA, U.S.A.

T. J. Burr, New York State Agricultural Experiment Station, Geneva, NY, U.S.A.

W. Bushnell, University of Minnesota, St. Paul, MN, U.S.A.

T. Charles, McGill University, Ste-Anne-de-Bellevue, Canada

P. Christie, University of Texas Health Science Center, Houston, TX, U.S.A.

V. Citovsky, State University of New York, Stony Brook, NY, U.S.A.

D. Cook, Texas A\&M University, College Station, TX, U.S.A.

S. Covert, University of Georgia, Athens, GA, U.S.A.

D. Cullen, University of Wisconsin, Madison, WI, U.S.A.

M. Daniels, John Innes Centre, Norwich, England

A. Das, University of Minnesota, St. Paul, MN, U.S.A.

W. Dawson, Citrus Research \& Education Center, Lake Alfred, FL, U.S.A.

P. De Wit, Wageningen Agricultural University, Wageningen,
The Netherlands

R. Dean, Clemson University, Clemson, SC, U.S.A.

G. DeLorenzo, University of Rome "La Sapienza," Rome, Italy

J. Denarie, INRA, Castanet-Tolosan, France

Y. Dessaux, CNRS Institut Science Vegetale, Gif-sur-Yvette, France

M. Dickman, University of Nebraska, Lincoln, NE, U.S.A.

R. Dickstein, Drexel University, Philadelphia, PA, U.S.A.

R. Dixon, Samuel Roberts Noble Foundation, Inc., Ardmore, OK, U.S.A.

M. Djordjevic, The Austrlaian National University, Canberra, Australia

J. M. Dow, John Innes Centre, Norwich, England

A. Drenth, University of Queensland, Brisbane, Australia

L. Dunkle, Purdue University, West Lafayette, IN, U.S.A.

K. Dunn, Boston College, Chestnut Hills, MA, U.S.A.

J. English, John Innes Centre, Norwich, England

M. Etzler, University of California, Davis, CA, U.S.A.

R. S. S. Fraser, Littlehampton, West Sussex, England

D. W. Gabriel, University of Florida, Gainesville, FL, U.S.A.

T. Gaffney, Ciba Biotechnology, Research Triangle Park, ND, U.S.A.

D. Gilchrist, University of California, Davis, CA, U.S.A.

L. Glass, University of British Columbia, Vancoucer, BC, Canada

R. Goldbach, Wageningen Agricultural University, Wageningen, The Netherlands

J. Golden, Texas A\&M University, College Station, TX, U.S.A.

C. Gonzalez, Texas A\&M University, College Station, TX, U.S.A.

S. Goodwin, Purdue University, West Lafayette, IN, U.S.A.

T. Grodon, University of California, Berkeley, CA, U.S.A.

P. Gresshoff, The University of Tennessee, Knoxville, TN, U.S.A.

D. Gross, Washington State University, Pullman, WA, U.S.A.

M. L. Guerinot, Dartmouth College, Hanover, NH, U.S.A.

D. Haas, Universite de Lausanne, Laysanne-Dorigney, Switzerland

M. G. Hahn, University of Georgia, Riverbend, GA, U.S.A.

J. Hamer, Purdue University, Lafayette, IN, U.S.A.

R. Hammerschmidt, Michigan State University, East Lansing, MI, U.S.A.

J. Harbinson, ATO-DLO, Wageningen, The Netherlands

D. J. Hassett, University of Cincinnati College of Medicine, Cincinnati, OH, U.S.A.

S. Y. He, Michigan State University, East Lansing, MI, U.S.A. M. Heath, University of Toronto, Toronto, ON, Canada 
C. Hemenway, North Carolina State University, Raleigh, NC, U.S.A.

A. Hirsch, University of California, Los Angeles, CA, U.S.A.

B. Hohn, Friederich Miescher Institut, Basel, Switzerland

R. Howard, DuPont Company, Wilmington, DE, U.S.A.

R. Hull, John Innes Centre, Colney, Norwich, United Kingdom

R. S. Hussey, University of Georgia, Athens, GA, U.S.A.

S. W. Hutcheson, University of Maryland, College Park, MD, U.S.A.

R. Innes, Indiana University, Bloomington, IN, U.S.A.

J. A. G. Irwin, University of Queensland, St. Lucia, Brisbane, Queensland, Australia

T. Jack, Dartmouth College, Hanover, NH, U.S.A.

A. Jackson, University of California, Berkeley, CA, U.S.A.

N. Keller, Texas A\&M University, College Station, TX, U.S.A.

H. H. Keyser, University of Hawaii, Paia, HI, U.S.A.

H. C. Kistler, University of Florida, Gainesville, FL, U.S.A.

W. Koeller, New York State Agricultural Experiment Station, Geneva, NY, U.S.A.

R. Koenig, Institut fur Krankenheit der Pflanzen, Braunschweig, Germany

E. Kondorosi, CNRS-Institut des Sciences Végétales, Gif-surYvette, France

H. Kouchi, National Institute of Agrobiological Resources, Tsukuba, Ibaraki, Japan

G. Lacy, Virginia Polytechnic Institute and State University, Blacksburg, VA, U.S.A.

M. Lagrimini, Ohio State University, Columbus, OH, U.S.A.

M. Lapidot, Institute of Plant Protection, The Volcani Center, Bet Dagan, Israel

M. Law, Ciba Biotechnology, Research Triangle Park, NC, U.S.A.

K. Lawton, Ciba Biotechnology, Research Triangle Park, NC, U.S. A

H. Leung, Washington State University, Pullman, WA, U. S. A

M. Levy, Purdue University, West Lafayette, IN, U. S. A.

C. H. Liao, Eastern Regional Research Center, Philadelphia, PA, U.S.A.

S. Lindow, University of Claifornia, Berkeley, CA, U. S. A

K. Lindstrom, University of Helsinki, Vikinkaari, Finland

G. Lomonossoff, John Innes Centre, Norwich, United Kingdom

S. Long, Stanford University, Stanford, CA, U. S. A

J. Lorang, Oregon State University, Corvallis, OR, U. S. A

M. Lorito, Universita Degli Studi Di Napoli "Federico II," Portici, Italy

S. Mackenzie, Purdue University, West Lafayette, IN, U.S.A.

A. Matthysse, University of North Carolina, Chapel Hill, NC, U. S. A.

A. Matzk, Institute for Biochemistry and Plant Virology, Braunschweig, Germany

M. Mayo, Scottish Crop Research Institute, Invergowie, Dundee, Scotland

M. Mazzola, USDA-ARS, Wenatchee, WA, U.S.A.

M. McClure, University of Arizona, Tucson, AZ, U.S.A.

B. McDonald, Texas A\&M University, College Station, TX, U.S.A.

J. McDowell, University of North Carolina, Chapel Hill, NC,
U.S.A.

J. McEvoy, University of Wisconsin, Madison, WI, U.S.A.

M. C. Metzler, University of Turku, Turku, Finland

R. Meyer, University of Texas, Austin, TX, U.S.A.

R. Michelmore, University of California, Davis, CA, U.S.A.

M. Milgroom, Cornell University, Ithaca, NY, U.S.A.

W. A. Miller, Iowa State University, Ames, IA, U.S.A.

K. Miller, The Pennsylvania State University, University Park, PA, U.S.A.

K. Minamisawa, Tohoku University, Katahira, Aoba-Ku, Japan

B. Mullin, The University of Tennessee, Knoxville, TN, U.S.A.

R. Nelson, Samuel Roberts Noble Foundation, Ardmore, OK, U.S.A.

G. Nester, University of Washington, Seattle, WA, U.S.A.

K. K. Nielsen, Danisco Biotechnology, Copenhagem, Denmark

D. Noel, Marquette University, Milwaukee, WI, U.S.A.

M. O'Brien, State University of New York at Buffalo, Buffalo, NY, U.S.A.

R. Oliver, University of East Anglia, Norwich, England

C. Opperman, North Carolina State University, Raleigh, NC, U.S.A.

B. Ownley, The University of Tennessee, Knoxville, TN, U.S.A.

D. Panaccione, West Virginia University, Morgantown, WV, U.S.A.

G. Payne, North Carolina State University, Raleigh, NC, U.S.A.

N. K. Peters, University of Tromso, Tromso, Norway

D. Phillips, University of California, Davis, CA, U.S.A.

M. L Pierce, Oklahoma State University, Stillwater, OK, U.S.A.

L. S. Pierson III, University of Arizona, Tucson, AZ, U.S.A.

J. Pitkin, Michigan State University, East Lansing, MI, U.S.A.

G. K. Podila, Michigan Technological University, Houghton, MI, U.S.A.

F. Ponz, Centro de Investigaciones y Tecnologia (CIT-INIA), Autopista, Madrid, Spain

U. B Priefer, RWTH Aachen, Botanische Institut, Deutschland, Germany

S. Pueppke, University of Missouri, Columbia, MO, U.S.A.

N. Quigley, University of Tennessee, Knoxville, TN, U.S.A.

P. Ratet, Institut des Sciences Végétales, Gif-Sur-Yvette Cedex, France

E. Rikkerink, Institute of New Zealand Ltd, Mt. Albert Research Centre, Auckland, New Zealand

P. Ronald, University of California, Davis, CA, U.S.A.

F. Salamini, Max-Planck-Institut fur Zuchtungsforshung, Germany

J. Salmeron, Ciba-Geigy Corporation, Research Triangle Park, NC, U.S.A.

R. Sandrock, Cornell University, Ithaca, NY, U.S.A.

S. Santa Cruze, Scottish Crop Research Institute, Invergowie, Dundee, Scotland

W. Schaefer, Botanical Institut, AMP 111, Hamburg, Germany

M. A. Schell, University of Georgia, Athens, GA, U.S.A.

J. Scott-Craig, Michigan State University, East Lansing, MI, U.S.A.

N. Shibuya, National Institute of Agrobiological Resources, 
Tsukuba, Ibaraki, Japan

H. Spaink, Leiden University, Leiden, The Netherlands

S. E. Stevens, Jr., University of Memphis, Memphis, TN, U.S.A.

D. Straney, University of Maryland, College Park, MD, U.S.A.

J. Streeter, Ohio Agricultural R\&D Center, Wooster, $\mathrm{OH}$, U.S.A.

R. H. Symons, The University of Adelaide, Glen Osmond, South Australia

E. Tacke, Bioplant, Bruggerfeld, Germany

N. Talbot, University of Exeter, Exeter, United Kingdom

I. Taylor, Horticulture Research Institute, Wellesbourne, Warwick, United Kingdom

F. Trail, Michigan State University, East Lansing, MI, U.S.A.

B. Tyler, University of California-Davis, Davis, CA, U.S.A.

C. P. Vance, University of Minnesota, St. Paul, MN, U.S.A.

K. A. Vandenbosch, Texas A\&M University, College Station, TX, U.S.A.
J. Vanderleyden, Katholieke University Leuven, Heverlee, Belgium

J. Verchot, John Innes Institute, Norwich, United Kingdom

D. P. S. Verma, The Ohio State University, Columbis, OH, U.S.A.

B. Vernooij, Ciba Agricultural Biotechnology, Research Triangle Park, NC, U.S.A.

A. Vivian, University of the West of England, Bristol, United Kingdom

G. Walker, MIT, Cambridge, MA, U.S.A.

J. G. H. Wessels, University of Groningen, Haren, Netherlands

S. Whisson, University of Queensland, Australia

F. White, Kansas State University, Manhattan, KS, U.S.A.

T. M. A. Wilson, Scottish Crop Research Institute, Invergowie, Dundee, Scotland

S. Winans, Cornell University, Ithaca, NY, U.S.A.

J. Yoder, University of California, Davis, CA, U.S.A.

O. Yoder, Cornell University, Ithaca, NY, U.S.A. 